\title{
LOS DESASTRES RECURRENTES EN MÉXICO: EL HURACÁN PAULINE Y LA TORMENTA MANUEL EN ACAPULCO, GUERRERO
}

\section{Frequent Disasters in México: Hurricanes Pauline and Manuel in Acapulco, Guerrero}

\section{Os Desastres Recorrentes no México: O Furacão Pauline e a Tormenta Manuel em Acapulco, Guerrero}

Juan Manuel Rodríguez Esteves, El Colegio de la Frontera Norte (México) jesteves@colef.mx

\section{Recibido: 12 de abril de 2016}

\section{RESUMEN}

Los huracanes y otras tormentas tropicales son fenómenos naturales que atraen el interés de las personas en todo el mundo, en especial cuando afectan comunidades costeras. Cada año, sobre todo durante la temporada de huracanes, es común leer o ver en los diferentes medios de comunicación los daños provocados por las tormentas tropicales en varios países, ante todo en América Latina y Asia.

Para el caso de México, el total de pérdidas económicas asociadas a fenómenos naturales ha ido en aumento. Durante el año 2000 se destinaron 230 millones de dólares (US) para la reconstrucción de la infraestructura afectada por fenómenos hidrometeorológicos, mientras que en el 2013 los daños ascendieron a 4476 millones de dólares, con un pico durante 2010 cuando se registraron 7208 millones de dólares en pérdidas. Por otro lado, del 
total de daños provocados por fenómenos naturales, el 92 \% estuvo asociado a fenómenos hidrometeorológicos, entre los que se encuentran los huracanes y otros fenómenos (SEGOB, 2014).

El objetivo de este artículo es analizar los impactos provocados por los desastres asociados a la influencia de los huracanes desde una perspectiva comparativa entre dos fenómenos en particular, el huracán Pauline en 1997 y la tormenta Manuel en 2013, eventos hidrometeorológicos que afectaron el estado mexicano de Guerrero, pero en especial el puerto de Acapulco. Una de las conclusiones principales de este estudio se refiere a que no importa solamente la intensidad del fenómeno natural para generar daños en la sociedad, sino que el total de daños también se refiere a los contextos de vulnerabilidad que una sociedad genera con el transcurso de los años.

Palabras clave: desastres, huracanes, México.

\section{ABSTRACT}

Hurricanes and other tropical storms are natural phenomena that attract the interest of people all over the world, especially when they affect coastal communities. Each year, especially during the hurricane season, it is common to read or see in the different media damage caused by tropical storms in several countries, especially in Latin America and Asia.

In Mexico total economic losses associated with natural phenomena has been increasing. During the year 2000 were allocated 230 million US dollars for the reconstruction of the infrastructure affected by hydrometeorological phenomena, while in 2013 damage amounted to \$ 4,476 million, peaking during 2010 were recorded when 7,208 million dollars in losses. On the other hand, the total of damage caused by natural phenomena, $92 \%$ were associated with hydrometeorological phenomena, which include hurricanes and other phenomena (SEGOB, 2014).

The aim of this paper is to analyze the impacts caused by disasters associated with the influence of hurricanes from a comparative perspective between two phenomena in particular, Hurricane Pauline in 1997 and Manuel storm in 2013 events hydrometeorological which affected the Mexican State of Guerrero, but especially to the port of Acapulco. One of the main conclusions of this study refers to that no matter only the intensity of the natural phenomenon to generate damage on society, but the total of damages also refers to the contexts of vulnerability generated by a society with the course of the years.

Keywords: Disasters, hurricanes, Mexico.

\section{RESUMO}

Os furacões e outras tormentas tropicais são fenômenos naturais que atraem o interesse das pessoas em todo o mundo, em especial quando estes afetam comunidades costeiras. Cada ano, especialmente durante a temporada de furacões, é comum ler ou ver nos diferentes meios de comunicação os danos provocados pelas tormentas tropicais em vários países, especialmente na América Latina e na Ásia. 
Para o caso do México, o total de perdas económicas associadas a fenômenos naturais tem ido em aumento. Durante o ano 2000 destinaram-se 230 milhões de dólares (US) para a reconstrução da infraestrutura afetada por fenômenos hidrometeorológicos, enquanto que no 2013 os danos ascenderam a 4,476 milhões de dólares, com um pico durante o 2010 quando se registraram 7,208 milhões de dólares em perdas. Por outro lado, do total de danos provocados por fenômenos naturais, o 92 \% estiveram associados a fenômenos hidrometeorológicos, entre os que se encontram os furacões e outros fenômenos (SEGOB, 2014).

O objetivo deste artigo é analisar os impactos provocados pelos desastres associados à influência dos furacões desde uma perspectiva comparativa entre dois fenômenos em particular, o furacão Pauline em 1997 e a tormenta Manuel no ano 2013, eventos hidrometeorológicos que afetaram o estado mexicano de Guerrero, mas especialmente ao porto de Acapulco. Uma das conclusões principais deste estudo refere-se a que não só importa a intensidade do fenômeno natural para gerar danos na sociedade, mas também que o total de danos também se refere aos contextos de vulnerabilidade que uma sociedade gera com o transcurso dos anos.

Palavras-chave: desastres, furacões, México.

\section{Amenaza, vulnerabilidad y desastres}

Los desastres asociados a fenómenos naturales en el mundo representan un importante desafío para los países están más expuestos a dichos fenómenos. El impacto que provocan se puede medir en términos de pérdidas de vidas humanas, personas heridas, viviendas e infraestructura destruidas o pérdidas económicas totales. Debido al aumento de los daños que provocan, algunos expertos señalan que se debe a un incremento en la intensidad de las tormentas, huracanes, sequías, inundaciones, etc. (perspectiva tecnocrática). Sin embargo, hay otros expertos que señalan que este incremento se debe no solo a la intensidad del fenómeno natural que los desencadena sino, principalmente, a la construcción social del riesgo cuyos factores están relacionados con el aumento de la vulnerabilidad social y con la exposición de las comunidades a dichos fenómenos (perspectiva de la vulnerabilidad). En esta última perspectiva se ubica el presente estudio.

Los fenómenos hidrometeorológicos intensos representan la amenaza más importante al nivel mundial en términos de pérdidas humanas y económicas. Para el caso de México, durante el año de 2013, los daños asociados a este tipo de fenómenos alcanzó el $92 \%$ del total de daños y pérdidas por fenómenos naturales, representando con ello la amenaza natural más importante que se debe atender en el corto plazo (SEGOB, 2014). Sin embargo, no solo la exposición a un fenómeno natural intenso está asociada directamente a la ocurrencia de un desastre. Existen tres elementos básicos que estrechamente relacionados entre sí y es condición necesaria que se combinen para que se manifieste un desastre.

En primer lugar se tiene a la amenaza, la cual es uno de los elementos centrales en la manifestación de un desastre. Esta investigación está relacionada con las amenazas asociadas a fenómenos naturales y, en particular, 
con los huracanes y tormentas tropicales. La amenaza puede ser definida como un fenómeno (natural o antrópico) cuya manifestación en un lugar y momento dado puede representar un potencial de daño a una sociedad o a una comunidad vulnerable a ella (Cardona, 1996). De acuerdo con el autor se puede entender que no cualquier fenómeno natural puede ser considerado una amenaza. El fenómeno debe alcanzar cierto umbral que represente el daño potencial a la comunidad expuesta al fenómeno.

Un ejemplo puede ser una lluvia de $50 \mathrm{~mm}$ en un periodo de 24 horas que se presenta en una región tropical como en la que se encuentra el sur de México, la cual puede pasar desapercibida por la mayor parte de la población. Pero si esa lluvia se presenta en otras latitudes donde las lluvias son escasas, característica de las regiones semiáridas al norte de México, muy probablemente la lluvia puede provocar daños de consideración, sobre todo si la población está asentada en zonas establecidas como de alto riesgo y ha desarrollado a través de los años evidencias de vulnerabilidad social hacia ese fenómeno.

La amenaza es con frecuencia confundida al ser considerada sinónimo de riesgo. Para algunos autores el término de amenaza hace referencia al peligro latente o potencial que puede representar una amplia variedad de fenómenos naturales como los terremotos, huracanes, erupciones volcánicas explosivas, etc. o de fenómenos asociados a la actividad humana como explosiones, accidentes industriales, terrorismo, guerras, etc. (Cardona, 1996). Sin embargo, cada vez es más común que se manifiesten desastres asociados a la combinación de fenómenos naturales y de origen humano o antrópicos, tal es el caso de las inundaciones, que se presentan en ciertos lugares y que están relacionadas con lluvias intensas (fenómeno natural), pero que se combinan con un mal manejo del territorio como pueden ser asentamientos urbanos mal planeados (fenómeno social).

Por su parte, algunos autores consideran la amenaza como una probabilidad de que ocurra un riesgo frente al cual una comunidad en particular es vulnerable (Wilches-Chaux, 1993). En este punto la amenaza presenta una connotación cuantitativa al ser necesario calcular una probabilidad de que cierto fenómeno intenso pueda provocar algún daño a una comunidad en particular. En cualquiera de las dos formas de concebir a la amenaza, lo importante es que ciertos fenómenos, ya sea de origen natural o antrópico, pueden representar un peligro o amenaza a cierta sociedad o comunidad. Por lo tanto, no todas las comunidades o sectores de la sociedad son igualmente vulnerables a cierto tipo de amenaza.

En lo que respecta al riesgo, exclusivamente al asociado a los desastres, de acuerdo con Cardona (1996), "puede ser el resultado de relacionar la amenaza, o probabilidad de ocurrencia de un evento, con la vulnerabilidad o susceptibilidad de los elementos expuestos. Algunas medidas de protección, como la utilización de tecnologías adecuadas no vulnerables, y otras como la regulación de usos del suelo y la protección del medio ambiente, son la base fundamental para reducir las consecuencias de las amenazas o peligros naturales y tecnológicos". Como se puede observar, existen definiciones un tanto contradictorias donde se mezclan tanto amenaza como riesgo. Por tal motivo, se considera que el riesgo es la probabilidad de daños y pérdidas que tengan un significado social, es decir, que sean reconocido por un amplio sector de la sociedad. El riesgo existe en la medida en que interactúen o se interrelacionen factores de amenaza y de vulnerabilidad (Lavell, 2002). Como se puede distinguir, tanto la amenaza como el riesgo son conceptos estrechamente relacionados, la presencia de uno obliga a la influencia del otro en un estado de mutua dependencia. Sin embargo, el concepto de vulnerabilidad es quizás el que menos confusión provoca de los ya mencionados, su importancia es central para entender los desastres. 
La vulnerabilidad puede ser entendida como la propensión de una sociedad o comunidad a sufrir daños por la presencia de un agente perturbador, en este caso representado por una amenaza en particular. Esto significa que no todas las sociedades o comunidades son vulnerables a todos los fenómenos, ya sean naturales o antrópicos, sino que pueden existir amenazas a las que sí son vulnerables y amenazas ante las que presenten grados menores de vulnerabilidad.

Para Wilches-Chaux (1993) la "vulnerabilidad está referida a la incapacidad de una comunidad para absorber, mediante el autoajuste, los efectos de un determinado cambio en su medio ambiente, o sea, su inflexibilidad o incapacidad para adaptarse a ese cambio, que para la comunidad constituye un riesgo". La vulnerabilidad, por lo tanto, deberá ser entendida como un contexto cambiante y dinámico debido a que desarrolla debilidades que se manifiestan de manera particular al momento en que se presenta una amenaza. Estas debilidades o incapacidades están asociadas a las llamadas dimensiones de la vulnerabilidad, las cuales engloban el aspecto físico, social, económico, ambiental, político, cultural, etc., de una sociedad específica (Cardona, 2001). Un elemento directamente relacionado con el término de vulnerabilidad es el de resiliencia, o la capacidad de adaptarse al cambio una vez que una comunidad ha sido afectada por una amenaza, el cual esté siendo considerado en los estudios cuya intensión es reducir los contextos de vulnerabilidad y aumentar las capacidades de respuesta de la sociedad hacia los desastres, es decir, aumentar la seguridad de los asentamientos humanos.

Con base en lo anterior, el desastre puede ser entendido como el momento en el que concurre una amenaza particularmente peligrosa a una comunidad, y la vulnerabilidad que manifiesta esa comunidad al momento de ocurrir la amenaza. De manera general, es relativamente fácil definir un desastre cuando se refiere a situaciones específicas en las que se manifiesta un fenómeno natural intenso y una comunidad vulnerable a ese fenómeno. Los daños de estos desastres se pueden observar en el número de fallecimientos, heridos, viviendas destruidas y sobre todo en la reducción del nivel de desarrollo de los países, en especial de los llamados economías emergentes, cuando la reconstrucción obliga a los gobiernos a destinar una buena parte de sus presupuestos anuales a atender los daños ocasionados por los fenómenos intensos. Por lo tanto, el desastre es una función entre la amenaza y la vulnerabilidad, pero debe entenderse que no todas las sociedad son igual de vulnerables que las otras, como se señaló antes. De manera general, el desastre puede ser explicado con la siguiente expresión.

\section{Desastre $=\mathrm{f}($ Amenaza $)($ Vulnerabilidad $)$}

Para establecer que no solamente basta con que una sociedad esté expuesta a una amenaza para que se manifieste un desastre, debe existir una vulnerabilidad a esa amenaza. De manera más amplia, Moseley (1999) pone un tema de discusión denominado catástrofes convergentes para indicar la presencia frecuente de "desastres naturales" en una misma región. Aquí, el término de catástrofe o desastre convergente puede ser comparado con la presencia de una enfermedad también recurrente en una región en particular. El autor demuestra que los desastres son provocados por la vulnerabilidad diferencial de las sociedades y no solo a partir de la amenaza. Para demostrar lo anterior, Moseley basa su estudio desde la perspectiva de la geoantropología y toma como ejemplo los impactos de El Niño y otras amenazas en la región andina (Moseley, 1999). Esta perspectiva de análisis es una herramienta importante para conocer y analizar cómo una sociedad puede verse afectada por la influencia de fenómenos naturales y sus consecuentes impactos al no reducir su vulnerabilidad, como es el caso de Acapulco, México. 


\section{Acapulco, Guerrero, México}

Por su ubicación geográfica y poseer costas tanto en el Golfo de México como en el Océano Pacífico, México se encuentra expuesto a la influencia de los ciclones tropicales, fenómenos que se caracterizan por producir fuertes vientos, lluvias intensas y alto oleaje. Los ciclones tropicales se presentan año tras año afectando a la población que se asienta próxima a las costas y, muchas veces, también a asentamientos lejanos a ellas. Para la temporada de huracanes de 2015, se presentaron 18 huracanes en el Océano Pacífico y 11 en el Océano Atlántico, Golfo de México y mar Caribe, algunos de estos fenómenos tuvieron influencia en territorio mexicano, lo que demuestra la gran exposición del país a estos (CONAGUA, 2016).

El estado de Guerrero, en la costa sur del Pacífico mexicano, ha sido afectado por un número significativo de tormentas tropicales en los últimos años. Guerrero es uno de los estados con una considerable actividad turística al contar con centros turísticos de importancia nacional e internacional, tal es el caso Ixtapa-Zihuatanejo y del puerto de Acapulco. Este ha sido un gran centro de desarrollo turístico desde la década de 1950, cuando los gobiernos de ese entonces vieron el potencial esa región. Esto dio como resultado que Acapulco fuera desde ese entonces una de las principales fuentes económicas para el estado de Guerrero y para el país en este sector. De esta forma el crecimiento poblacional del municipio de Acapulco experimentó un fuerte aumento a partir de las décadas del cincuenta y sesenta, llegando a una población de 673479 habitantes para el año 2010 (ver tabla 1).

\section{Tabla 1. Población total del municipio de Acapulco para diferentes años}

\begin{tabular}{|l|l|}
\hline \multicolumn{1}{|c|}{ Año } & \multicolumn{1}{|c|}{ Población total } \\
\hline 1900 & 4932 \\
\hline 1921 & 5768 \\
\hline 1940 & 9993 \\
\hline 1960 & 49149 \\
\hline 1980 & 301902 \\
\hline 2000 & 620656 \\
\hline 2010 & 673479 \\
\hline
\end{tabular}

Fuente: INEGI, Marco Geoestadístico Nacional, consultado el 14 de marzo de 2016 en:

De acuerdo con la tabla 1, el crecimiento poblacional de Acapulco había tenido un crecimiento "lento" desde inicios del siglo XX y hasta la década del cuarenta, relacionado con la vocación pesquera y comercial que dio origen a este puerto. Pero gracias a su desarrollo como centro turístico a partir de la década de los cincuenta, Acapulco experimentó un crecimiento sostenido e intenso, el cual se registra hasta la actualidad. La exposición de una población en constante crecimiento a fenómenos naturales intensos como los huracanes ha traído como consecuencia la manifestación de desastres en varias ocasiones, y más en los últimos 40 años. Para el periodo de 1970 


\section{DISERTACIONES}

ESTUDIOS

a 2011, el estado de Guerrero ha sufrido el impacto directo de por lo menos 24 ciclones tropicales, destacando los años 1974 y 1996 cuando se presentaron tres ciclones en cada temporada (CONAGUA, 2012).

En lo que respecta a las condiciones naturales, el clima para el puerto de Acapulco es del tipo cálido subhúmedo y posee un régimen de lluvias en verano. Esta característica le ha conferido gran parte de su importancia turística ya sea para el turismo nacional como internacional. El promedio de precipitación anual para Acapulco es de 1337 mm, la distribución de la lluvia durante un año se presenta en la gráfica 1.

\section{Gráfica 1. Distribución de la precipitación normal mensual. \\ Estación Acapulco de Juárez (SMN)}

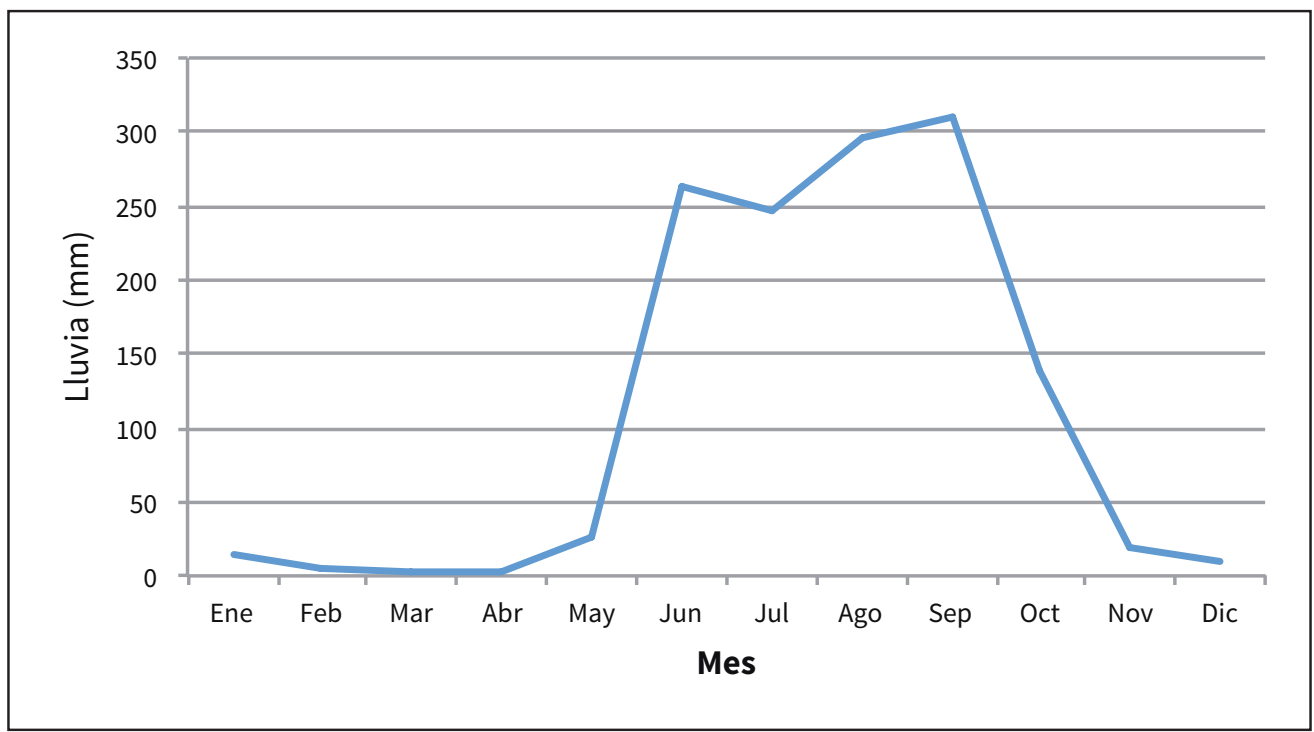

Fuente: Normales climatológicas. Estación Acapulco de Juárez (SMN), consultado el 6 de marzo de 2016, en: http://smn.cna.gob.mx/index.php?option=com_content\&view=article\&id=42\&ltemid=75

La mayor parte de las precipitaciones se presenta entre los meses de junio a octubre, lo cual coincide con la temporada de huracanes para el Océano Pacífico Norte (mayo a noviembre), destacando los meses de agosto y septiembre como los meses con mayores precipitaciones. Es precisamente en estos últimos meses de lluvias en los que los huracanes que han provocado un mayor costo se han presentado en Acapulco y en Guerrero en general; tal es el caso del huracán Pauline y la tormenta Manuel. Para el estudio de los desastres, es importante considerar los valores máximos de las variables hidrometeorológicas en periodos de tiempo corto como lo son 24 horas, como más adelante se discutirá. 


\section{Huracán Pauline (5-10 de octubre de 1997)}

En la mañana del 6 de octubre de 1997, la depresión tropical № 18-E se desarrolló a tormenta tropical y recibió el nombre de Pauline, la cual mantuvo vientos sostenidos de $75 \mathrm{~km} / \mathrm{h}$ y rachas de $90 \mathrm{~km} / \mathrm{h}$. En ese momento la tormenta se ubicó frente a las costas del Pacífico mexicano a $395 \mathrm{~km}$ al suroeste de Tapachula, Chiapas. Durante ese mismo día, a las 16:00 horas, Pauline cambió a huracán categoría 1 al alcanzar vientos sostenidos de $120 \mathrm{~km} / \mathrm{h}$ y rachas de $150 \mathrm{~km} / \mathrm{h}$ (CONAGUA, 2009).

Para la mañana del 8 de octubre, el huracán Pauline, ya de categoría 4, alcanzó vientos máximos de $210 \mathrm{~km} / \mathrm{h}$ y rachas de $260 \mathrm{~km} / \mathrm{h}$ ubicándose a $100 \mathrm{~km}$ al sur-suroeste de Huatulco, Oaxaca. En la tarde de ese día Pauline toca tierra entre las poblaciones de Puerto Ángel y Puerto Escondido, Oaxaca, pero con categoría 3, con vientos máximos de $185 \mathrm{~km} / \mathrm{h}$ y rachas de $240 \mathrm{~km} / \mathrm{h}$ (CONAGUA, 2009).

Una vez en tierra, el huracán Pauline se desplazó con una orientación paralelo a la línea de costa, con una trayectoria hacia el noroeste llegando al estado de Guerrero, por lo que el 9 de octubre el "ojo" del huracán llegó a $30 \mathrm{~km}$ al nor-noroeste del puerto de Acapulco, Guerrero, con vientos sostenidos de $165 \mathrm{~km} / \mathrm{h}$ y rachas de hasta $200 \mathrm{~km} / \mathrm{h}$. Las paredes de Pauline golpearon fuertemente el puerto de Acapulco provocando lluvias intensas por efecto de las montañas cercanas (CONAGUA, 2009). La trayectoria del huracán Pauline se presenta en la figura 1.

Figura 1. Trayectoria del huracán Pauline, 1997

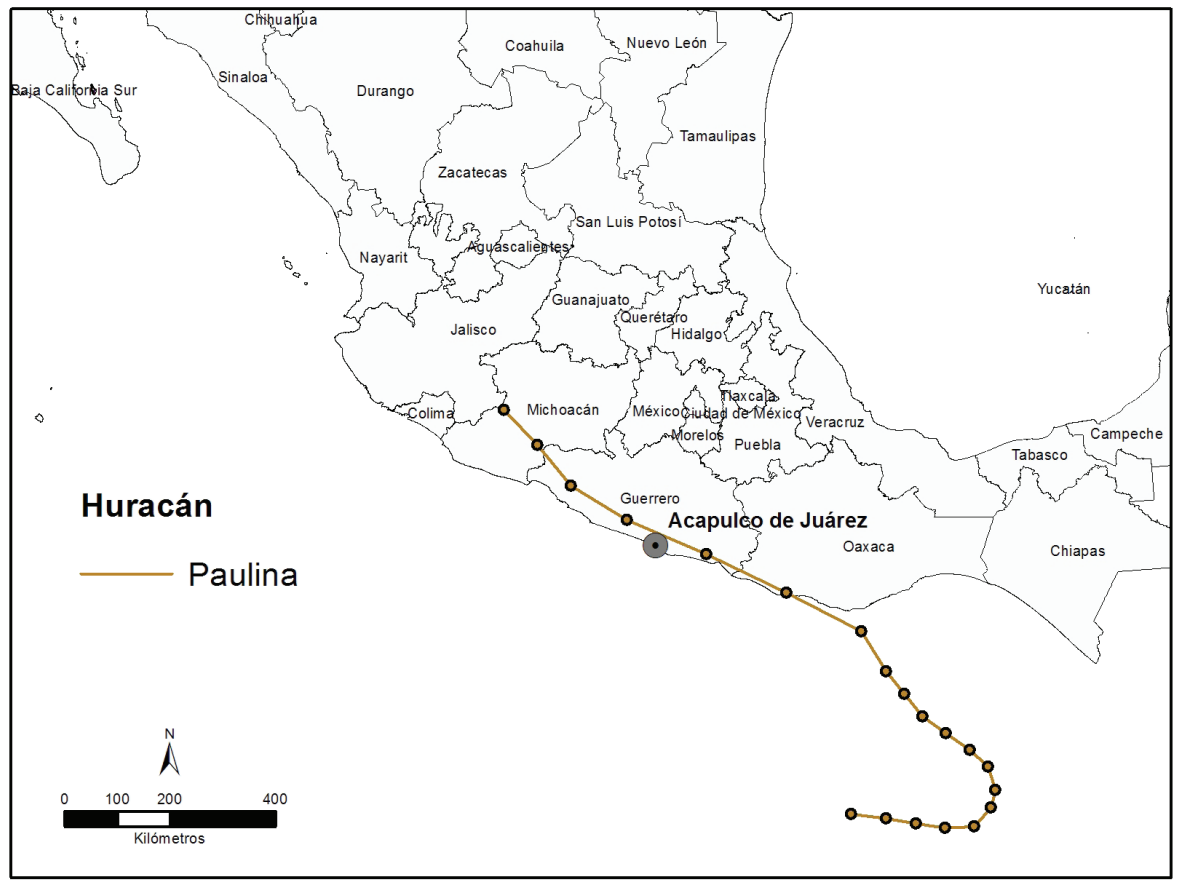

Fuente: elaboración propia con datos de ftp://eclipse.ncdc.noaa.gov/pub/ibtracs/v03r08/all/shp/year/ Consultado el 7 de marzo de 2016. 
El principal intento de informar a la población potencialmente expuesta al impacto de Pauline estuvo a cargo del Servicio Meteorológico Nacional (SMN), organismo oficial que monitorea y difunde información meteorológica para todo el país a través del sistema de avisos de ciclones tropicales. Durante la emergencia de Pauline el SMN emitió 32 avisos y dos boletines meteorológicos para mantener informada a la sociedad y a las dependencias del Gobierno Federal, Estatal y Municipal como medida preventiva y de protección a la población y a sus propiedades e infraestructura pública ante la inminente llegada de Pauline a las costas de México (CONAGUA, 1998). Los avisos son mensajes meteorológicos cuya intensión es alertar a la población ante el riesgo de llegada de un fenómeno hidrometeorológico, mientras que un boletín es un informe periódico que muestra las condiciones meteorológicas más recientes sobre la evolución del ciclón (SMN-CONAGUA, 2016). A pesar de contar con información detallada sobre este ciclón, la población de Acapulco, así como sus autoridades, no pudieron resistir los embates del huracán ya que se generó una gran cantidad de lluvia en un corto tiempo, la cual provocó repentinas crecidas de arroyos y ríos de toda la región.

Debido a la presencia de Pauline en las costas mexicanas se registraron lluvias intensas durante el mes de octubre de 1997, lo que resultó en que fue uno de los meses más húmedos desde la década del cuarenta. El volumen total de lluvia aportado por Pauline equivale a más de la mitad de la registrada durante todo el mes de octubre: en tan solo en un periodo de 24 horas hubo una precipitación acumulada de más de $400 \mathrm{~mm}$ (Matías, 1998).

Los días 8 y 9 de octubre de 1997 representaron para Acapulco los momentos más intensos en cuanto a la exposición de la sociedad a la amenaza natural que pueden representar las lluvias intensas. Como lo muestra la Gráfica 2, en la estación climatológica de Acapulco de Juárez, se presentó una lluvia acumulada de 405 mm durante ambos días, de los cuales $360 \mathrm{~mm}$ se registraron solo el día 8 de octubre. De manera comparativa, durante octubre de 1997 solo se habían registrado lluvias significativas los días 1 y 2 con lluvias acumuladas de tan solo 53,5 y 35,5 mm respectivamente.

Debido a las fuertes lluvias ocurridas en Acapulco, Guerrero, durante un periodo de tiempo relativamente corto, se registraron muertes y pérdidas económicas significativas, siendo este el mayor desastre en la región hasta el momento. Sin embargo, la historia de repetiría 16 años más tarde con el paso de la tormenta tropical Manuel en 2013. 
Gráfica 2. Precipitación acumulada en octubre de 1997.

Estación Acapulco de Juárez (SMN)

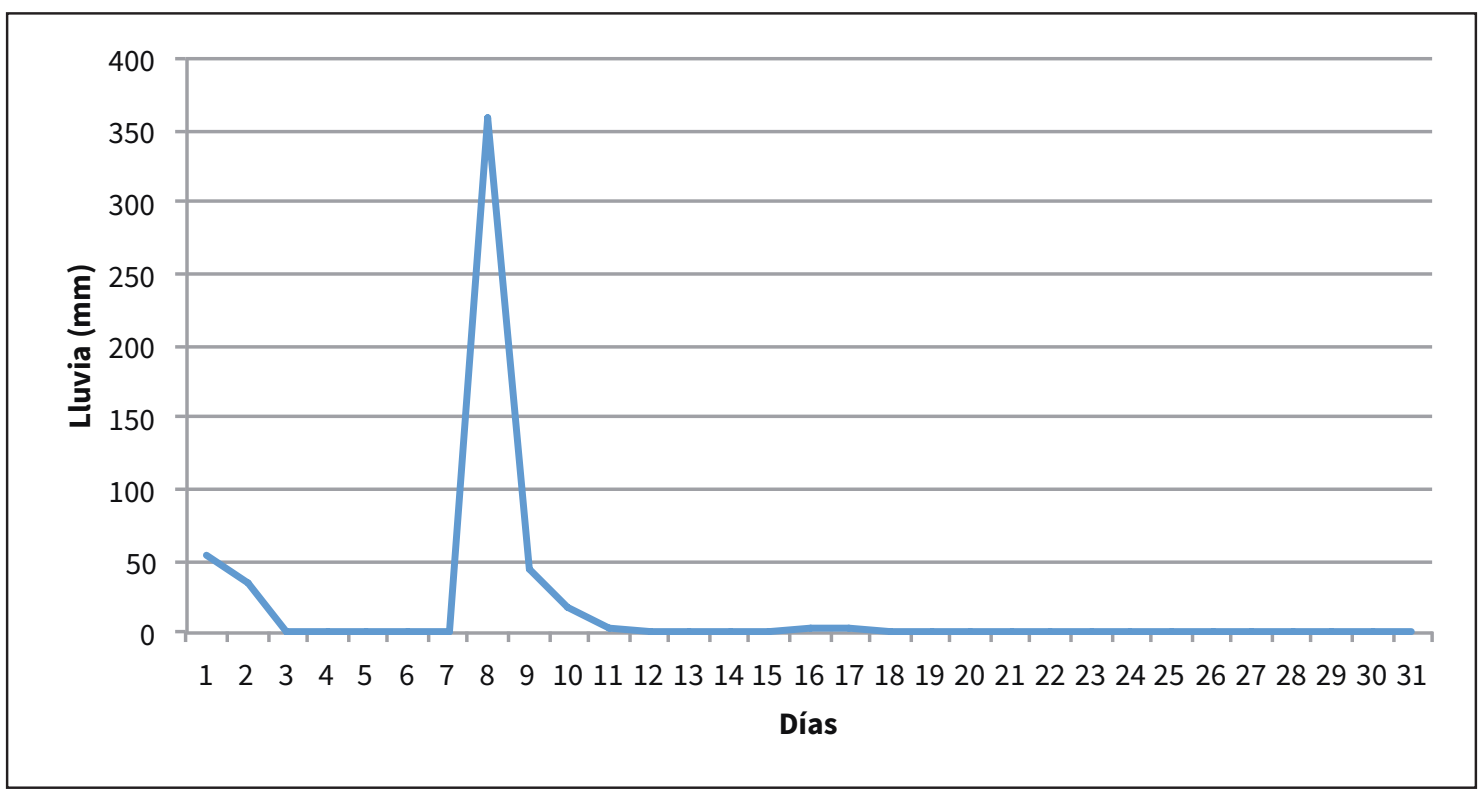

Fuente: Estación 12142. Acapulco de Juárez, Guerrero. CNA-SMN-SCDI Climatología estadística. Consultado el 6 de marzo de 2016 en: http://smn.conagua.gob.mx/index.php?option=com_content

\section{Tormenta Manuel (13-19 de septiembre de 2013)}

El día 13 de septiembre de 2013, por la mañana, se desarrolló la depresión tropical № 13 frente a las costas del estado de Guerrero, con vientos sostenidos de $55 \mathrm{~km} / \mathrm{h}$ y rachas de $75 \mathrm{~km} / \mathrm{h}$, y un desplazamiento hacia el noroeste a $6 \mathrm{~km} / \mathrm{h}$. Por la tarde de ese mismo día, se desarrolló la tormenta tropical Manuel con vientos sostenidos de $75 \mathrm{~km} / \mathrm{h}$ y rachas de $85 \mathrm{~km} / \mathrm{h}$, y un desplazamiento hacia el oeste-noroeste a una velocidad de $9 \mathrm{~km} / \mathrm{h}$ (CONAGUA, 2013).

La tormenta tropical Manuel continúo su progreso y el día 14 de septiembre se dirigió hacia el norte mientras aumentaba su fuerza frente a las costas de Guerrero y Michoacán, originando una amplia zona de nubosidad densa y, por lo tanto, favoreciendo una entrada importante de humedad hacia los estados de Guerrero, Michoacán, Colima y Jalisco. Posteriormente, el día 15 de septiembre, Manuel tocó tierra en las cercanías del puerto de Manzanillo, Colima, con vientos sostenidos de $100 \mathrm{~km} / \mathrm{h}$ y rachas de $130 \mathrm{~km} / \mathrm{h}$, perdiendo fuerza mientras se internaba en el continente. A pesar de ello, la tormenta tropical provocó lluvias intensas en los estados de Jalisco 
y Colima; Manuel recobró fuerza cuando se internó de nuevo en el Océano Pacífico al cruzar el estado de Jalisco, llevando importantes flujos de humedad a los estados de Nayarit y Sinaloa. Finalmente, y ya como depresión tropical remanente, Manuel se degradó en el estado de Chihuahua el 19 de septiembre a las 22:00 horas (CONAGUA, 2013). La trayectoria de la tormenta Manuel se presenta en la figura 2.

Figura 2. Trayectoria de la tormenta Manuel, 2013

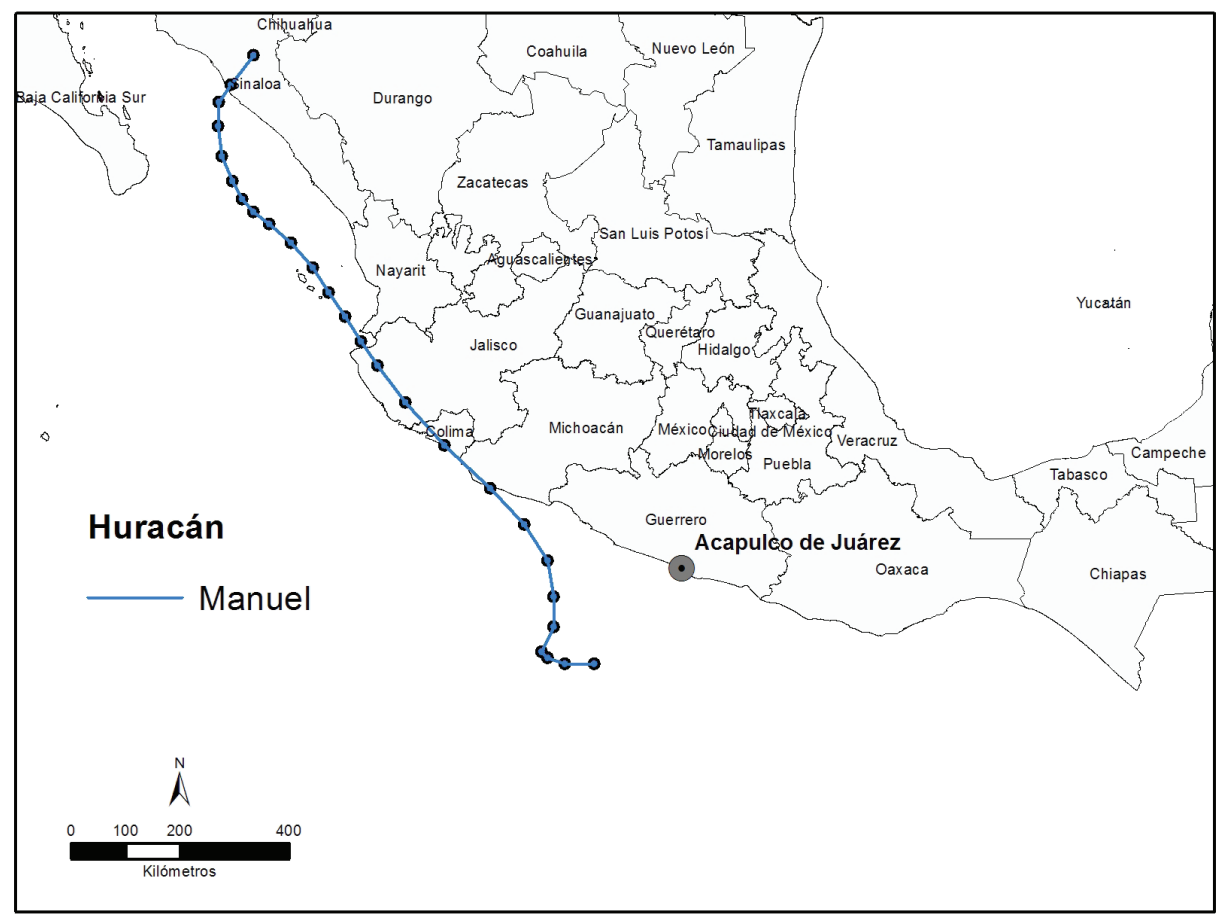

Fuente: elaboración propia con datos de ftp://eclipse.ncdc.noaa.gov/pub/ibtracs/v03r08/all/shp/year/ Consultado el 7 de marzo de 2016.

El Sistema Nacional de Protección Civil, dependiente de la Secretaría de Gobernación y con datos del Servicio Meteorológico Nacional emitió 42 avisos y boletines meteorológicos, además de la publicación en su portal electrónico de 28 vigilancias tropicales (CONAGUA, 2016c). El boletín de alerta por ciclón tropical № 02, que se emitió el 13 de septiembre a las 16:30, hora local, ya consideraba una alerta amarilla para el estado de Guerrero y el sur de Michoacán, con lo que se recomendaba revisar planes y procedimientos de comunicación y operación de las autoridades locales de protección civil (estatales y municipales) y se consideraba también la posibilidad de evacuación de la población (SEGOB, 2016).

La tormenta Manuel representó uno de los ciclones tropicales con mayores pérdidas económicas para México. Con base en datos del Centro Nacional de Prevención de Desastres (CENAPRED), las lluvias y vientos asociados a Manuel provocaron 111 muertes en todo el país, pero con influencia del huracán Íngrid, que se presentó de manera 
simultánea en la costa del Golfo de México. Por su parte, se registraron 1576 viviendas dañadas y 27 528,6 millones de pesos en pérdidas en varios estados del Pacífico mexicano (SEGOB, 2014).

La Gráfica 3 muestra la precipitación registrada durante el mes de septiembre de 2013. A diferencia de Pauline en octubre de 1997, Manuel afectó durante un mes en el que ya se habían registrado lluvias significativas, lo que agravó la situación de los habitantes de Acapulco al incrementarse los daños asociados a esta tormenta. De esta manera, las lluvias provocadas por Manuel alcanzaron los $432 \mathrm{~mm}$ durante los días 14 y 15 de septiembre de 2013, cuyos volúmenes fueron de 226 y $206 \mathrm{~mm}$ respectivamente. Sin embargo, la humedad asociada a Manuel ocurre en un momento en que el suelo se encontraba saturado debido a las lluvias registradas los días 1 y 2 de septiembre con 40 y $22 \mathrm{~mm}$ respectivamente, otra lluvia significativa el día 5 con $38 \mathrm{~mm}$ y finalmente los días 11 y 13 con lluvias acumuladas de 51 y $63 \mathrm{~mm}$ en 24 horas.

La presencia de lluvias en los días previos al paso de Manuel fue una condición importante para la conformación del desastre asociada a esta tormenta. Si bien la trayectoria de Manuel no cruzó por tierras de Guerrero, sí provocó que las lluvias previas saturaran el suelo, lo que permitió la alta escorrentía arrastrando todo a su paso al incrementarse los niveles de agua en los arroyos y ríos de la región, especialmente en las cuencas hidrográficas dentro del puerto de Acapulco.

El impacto de Manuel en Acapulco hizo recordar a la población local los daños provocados por Pauline 16 años atrás, lo que desencadenó una serie de reclamos de la sociedad hacia los representantes del Gobierno, tanto local como estatal, debido a la desinformación acerca de la severidad del fenómeno así como a la poca supervisión de los planes de desarrollo urbano y de la regulación del uso del suelo que permitieron la invasión de terrenos que no presentaban condiciones seguras, además de no contar con la infraestructura urbana necesaria. El reclamo popular se presentó en un contexto en el que se dio prioridad a la infraestructura estratégica, que en este caso se refería a la infraestructura turística, para evitar el agravamiento de la principal actividad económica de Acapulco.

\section{Discusión}

El impacto de las tormentas tropicales en la costa de Guerrero no es sinónimo de desastres en sí. Como se comentó líneas atrás, la amenaza de origen natural cobra relevancia cuando se manifiesta en un contexto en el que la población va construyendo escenarios de vulnerabilidad. Esa vulnerabilidad se traduce en situaciones que debilitan a una comunidad para hacer frente a un agente perturbador, por ejemplo, entre los factores que incrementan la vulnerabilidad se encuentra la ubicación de viviendas en zonas inundables sin las debidas medidas de prevención, de viviendas cercanas a los cauces de arroyos y ríos, la falta de cumplimiento de los códigos locales de construcción, la poca organización comunitaria, la falta de preparación de las autoridades para informar, el actuar y hacer planes de protección frente a este tipo de situaciones, etc. 
Gráfica 3. Precipitación acumulada en septiembre de 2013.

Estación Acapulco de Juárez (SMN)

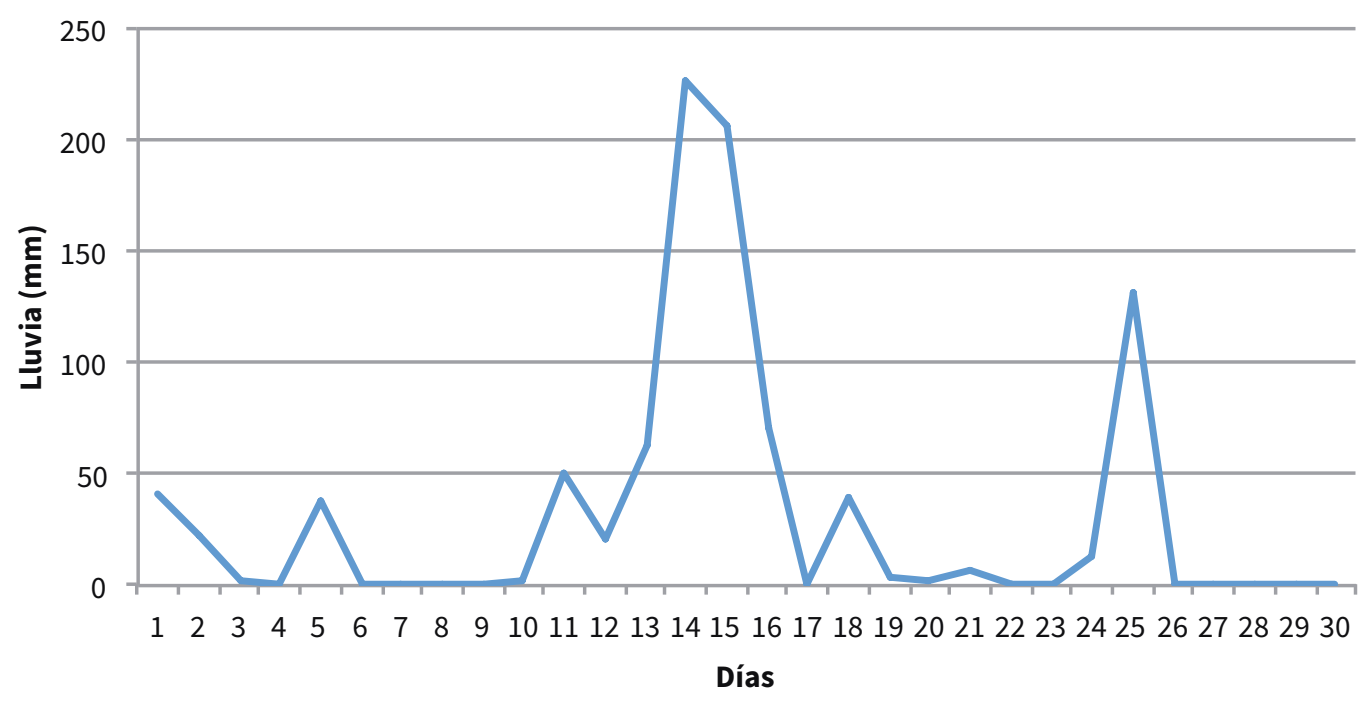

Fuente: Estación 12142. Acapulco de Juárez, Guerrero. CNA-SMN-SCDI Climatología estadística. Consultado el 6 de marzo de 2016 en: http://smn.conagua.gob.mx/index.php?option=com_content \&view=article\&id=182:guerrero\&catid=14:normales-por-estacion

El acelerado crecimiento demográfico del puerto de Acapulco, a partir del desarrollo turístico que se fomentó en esta región del Pacífico mexicano, fue un foco de atracción de importantes flujos migratorios procedentes de otros estados del país. Por sí mismo, el rápido crecimiento de la población no representa la ocurrencia de más desastres, pero sí es importante saber dónde se asienta una población que no puede acceder a una vivienda segura ante los efectos de las tormentas tropicales. Lo mismo ocurre con la infraestructura civil como carreteras, puentes, etc., cuya construcción no reúne los requisitos de seguridad para hacer frente a los fuertes vientos o crecida de arroyos o ríos.

Por su parte, el crecimiento urbano de Acapulco experimentó cambios significativos a partir de la década del cincuenta debido, en gran medida, a la construcción de la carretera Ciudad de México-Acapulco, que estimuló la llegada de más personas que se incorporaron a la actividad turística, lo que provocó que el crecimiento de la ciudad llegara a las partes más altas de las cuencas hidrográficas inmediatas del puerto. De esta forma, en 1960, la ciudad cubría una superficie de 1600 hectáreas, mientras que en 1980 la superficie alcanzó más de 3000 (Villegas, 2005, p. 164). En las décadas posteriores, los flujos migratorios que siguieron llegando a Acapulco provocaron que la zona urbana alcanzara las 4500 hectáreas en 1993 (SEDESOL, 2004), 6500 en 2003 (SEDESOL, 2004) y para el año 2015 alcanzó una superficie de 8500 hectáreas (DWUA, 2015). 
Este rápido crecimiento de la ciudad provocó que se anexaran a la superficie urbana terrenos que no cumplían con los requerimientos para el desarrollo urbano, tanto en términos técnicos (pendientes, cercanías a cauces de arroyos, etc.) como sociales (tenencia de la tierra, usos del suelo, entre otros), lo que aumentó de manera importante la exposición ante la amenaza de lluvias intensas y fuertes vientos provocados por los huracanes. Fue así como en 1997 la población más afectada por el huracán Pauline estaba ubicada en zonas de inundación o sobre cauces de arroyos que bajan de la sierra a la zona conocida como el Anfiteatro de Acapulco, en el centro de la ciudad, cuyos terrenos habían sido regularizados en años anteriores (Villegas, 2005, p. 171).

Un elemento del medio físico que está directamente relacionado con las inundaciones y la escorrentía provocadas por el huracán Pauline y la tormenta Manuel son las cuencas hidrográficas. El terreno sobre el cual se asienta Acapulco está conformado por planicies costeras (hacia el suroeste), piedemonte y sierras bajas (hacia el noreste), que tienen más de 900 metros sobre el nivel medio del mar (H. Ayuntamiento Constitucional de Acapulco de Juárez, 2015). Por su parte, la red de drenaje natural de Acapulco está conformada por cuencas hidrográficas de poca extensión, lo que provoca que en pocos minutos se presenten crecidas de arroyos e inundaciones de la partes bajas cuando se registran lluvias intensas como las que experimentó en 1997 y 2013. Las principales cuencas de la ciudad de Acapulco son:

Tabla 2. Superficie de cuencas hidrográficas de Acapulco, Guerrero

\begin{tabular}{|l|c|}
\hline \multicolumn{1}{|c|}{ Cuenca } & $\begin{array}{c}\text { Superficie } \\
\text { (hectáreas) }\end{array}$ \\
\hline Aguas Blancas & 960,5 \\
\hline Palma Sola Camarón & 1275,2 \\
\hline Magallanes & 317,6 \\
\hline La Garita & 739,4 \\
\hline Deportivo & 124,1 \\
\hline Costa azul & 764,7 \\
\hline Icacos & 373,9 \\
\hline Total & 4578,9 \\
\hline
\end{tabular}

Fuente: Plan de Desarrollo Urbano de la Ciudad de Acapulco, 1976, tomado de: Villegas (2005, p. 172).

El rápido aumento del nivel de agua en los arroyos y ríos tomó por "sorpresa" a muchos habitantes de Acapulco provocando el arrastre de viviendas, y destrucción y remoción en masa de materiales próximos a los cauces. Ante ello, la respuesta de las autoridades locales fue poca debido a la magnitud de los daños y a que no se contaba con protocolos de acción ante este tipo de eventos.

En lo que respecta a los daños asociados a el huracán Pauline y a la tormenta Manuel, se puede observar que existe un aumento en el monto total de pérdidas económicas que pueden ser cuantificables, es decir, aquellas que reportan la autoridad o las empresas aseguradoras. La tabla 3 muestra un resumen de las principales daños provocados por ambos fenómenos. 
Tabla 3. Principales daños asociados a Pauline y a Manuel en Acapulco, Guerrero

\begin{tabular}{|l|c|c|}
\hline \multicolumn{1}{|c|}{ Daños } & Huracán Pauline & Tormenta tropical Manuel \\
\hline Muertos & 102 & 169 \\
\hline Viviendas destruidas & 500 & 437 \\
\hline Damnificados & 9000 & 600 \\
\hline Evacuados & 10000 & 21767 \\
\hline Pérdidas económicas & 448 & 2270,00 \\
\hline Infraestructura carretera & (millones de US dólares) & (millones de US dólares) \\
\hline Turistas afectados & 11 cortes o derrumbes & 20 cortes o derrumbes \\
\hline $\begin{array}{l}\text { Lluvia acumulada en 24 y 48 horas } \\
\text { (mm) }\end{array}$ & No determinado & 40000 \\
\hline
\end{tabular}

Fuente: Elaboración propia con datos de Matías Ramírez (1998, p. 13); La Jornada, (1997); Bitrán, (2001); Emdat, (2016); SEGOB, (2013 y 2014); CONAGUA, (2016b).

Los datos que presenta la tabla 3 son en su mayoría datos oficiales, lo que puede ofrecer un panorama parcial de los daños debido a que son las instancias gubernamentales las encargadas de contabilizar y de dar respuesta a la sociedad afectada. Según otras fuentes consultadas, el número de muertos y de viviendas destruidas, por ejemplo, fuer mayor al reportado por las fuentes oficiales.

Un elemento que resalta en la tabla 3 es el número de muertos. En ambos eventos la suma de muertos fue mayor a 100, sin embargo Pauline estuvo prácticamente sobre el puerto de Acapulco, mientras que Manuel no tocó tierra en el estado de Guerrero, pero provocó un mayor volumen de lluvia en 24 y 48 horas. Este es un dato importante en el estudio de los desastres puesto que no es determinante el total de lluvia en un periodo de tiempo relativamente largo, es decir, una semana o un mes, pero es más determinante conocer el total de lluvia registrada en un periodo corto de tiempo como lo son 24 horas o en periodos más cortos. De esta forma, la precipitación registrada durante dos días consecutivos, incluso en un solo día, fue mayor durante Pauline que durante Manuel, pero el mayor número de fallecimientos se dio durante la segunda tormenta analizada, a pesar de que la lluvia acumulada en 48 horas fue mayor.

Por otro lado, las pérdidas económicas son otro elemento relacionado directamente con la magnitud de los daños en la zona de estudio. Las pérdidas registradas con el paso de Manuel casi quintuplicaron las registradas durante Pauline, a pesar de que durante Manuel se registraron menos lluvias diarias. Esto explica que en 2013 haya existido una mayor exposición de personas e infraestructura que con respecto a 1997 y, por lo tanto, mayor vulnerabilidad. En este sentido, Manuel provocó más de 900 millones de US dólares en daños en el sistema carretero de jurisdicción estatal, y más de 85 millones de US dólares en apoyos para la restauración de vivienda. Esta última cifra no representa el total de pérdidas asociadas a viviendas, debido a que el Estado mexicano, a través del Fondo Nacional de Desastres (FONDEN), solo proporciona apoyos a sectores estratégicos contenidos en las reglas de operación del fondo, por lo que un gran número de personas tuvo que realizar labores de reconstrucción sobre 
sus propias viviendas. Otros de los sectores estratégicos que más se vieron beneficiados con apoyos por parte de este fondo fueron el hidráulico y el escolar, en sus ámbitos estatales y federales.

Los apoyos federales y estatales para la reconstrucción de los daños provocados por Manuel no solamente se aplicaron durante 2013; el siguiente año se destinaron 1060 millones de US dólares en los mismos sectores mencionados, destacando el sector carretero estatal y el hidráulico federal. Debido al mes en el que se presentó Manuel, y a los cierres de los años fiscales para ejercer los recursos para la reconstrucción, durante 2014 se destinaron importantes recursos económicos para tratar de recuperar la infraestructura dañada o perdida. Sin embargo, la importancia de Acapulco para la actividad turística nacional e internacional fue un elemento sustancial para que los recursos que se aplicaron en 2013 siguieran llegando en 2014, lo que pone de manifiesto que el sector turístico es estratégico para México. Algunos casos similares se registraron en 2005 con el paso del huracán Vilma por el estado de Quintana Roo, y en Cancún particularmente, cuando se registraron importantes pérdidas en la infraestructura turística de ese destino turístico internacional.

Un dato importante respecto a la conformación urbana de una gran parte de la zona habitacional de Acapulco ha sido determinado, en buena medida, por formas espontáneas de ocupación del suelo, esto se manifiesta en el ámbito local al ocupar zonas con fuerte pendiente (mayores al $15 \%$ ), terrenos arenosos e inestables, terrenos en breña con vegetación que obstruye las construcciones, zonas propensas a inundaciones y terrenos de explotación agropecuaria (H. Ayuntamiento de Acapulco de Juárez, 2015).

A pesar de que en ambos eventos las autoridades federales emitieron información meteorológica de alerta tanto a los gobiernos estatales como a la población expuesta, la información no fue suficiente para evitar los desastres. Esto tiene relación con el proceso de la comunicación del riesgo que no se llevó de manera completa ni en 1997 ni en 2013. La comunicación del riesgo consta por lo menos de cinco fases que son: preparación, inicio, control, recuperación y evaluación (Presidencia de la Nación, 2016). Para los dos casos que aquí se analizan, solamente se cumplió con la etapa de elaboración de mensajes, canales y formatos de preparación para la preparación y respuesta, dejando de lado el resto de las etapas. Esto tiene significado porque ni las autoridades ni la población local estaban preparadas para responder a la emergencia lo que tuvo como resultado los contextos de desastre que se mencionaron arriba.

Para las fases de inicio, control, recuperación y evaluación de la comunicación del riesgo, se dieron acciones desarticuladas tomadas por las diferentes dependencias de Gobierno para atender la emergencia, no para evitarla. De esta forma, el Gobierno hizo esfuerzos por tratar de mitigar los daños de las inundaciones y atender, en la medida de lo posible, los efectos negativos de la emergencia atendiendo a los damnificados, llevando a cabo el desazolve y la limpieza de canales, remoción de escombros y la recuperación de la infraestructura urbana estratégica (rehabilitación de calles, puentes, etc.). Sin embargo, el proceso de reconstrucción llevó mucho tiempo ya que se trata de atender deficiencias urbanas y problemas estructurales no resueltos desde años atrás, por lo que se suman de nuevo a los contextos previos de vulnerabilidad, tanto física, social, como económica e institucional.

El paso del huracán Pauline y de la tormenta Manuel por Acapulco puede estar asociado a lo que Moseley (1999) denominó catástrofes convergentes. Esto se explica en la medida en que se conjugan en un espacio y tiempo determinado una sociedad que es vulnerable a una amenaza en particular, y por el otro, un fenómeno natural que se presenta con cierta regularidad afectando la vida cotidiana de las personas que viven en esas comunidades.

\section{8}


Si bien es cierto que los huracanes y otras tormentas tropicales son relativamente fáciles de pronosticar y monitorear, aún se presentan daños significativos en las comunidades expuestas a estos fenómenos. No obstante, la construcción de contextos de vulnerabilidad provoca que en ciertas regiones del mundo y en Latinoamérica, en particular, los daños sean cada vez más importantes debido al aumento del número de población y de infraestructura asentada en zonas consideradas de alto riesgo ante un gran número de amenazas asociadas a fenómenos naturales.

\section{Comentarios finales}

La presencia de tormentas tropicales, en general, y de huracanes, en particular, en las costas del Océano Pacífico, es una manifestación de la dinámica natural de los mares y la atmósfera. Esto le imprime a la costa mexicana características únicas que se manifiestan en la gran diversidad biológica típica de las zonas tropicales. Sin embargo, la presencia de comunidades próximas a las costas representan un desafío en la medida en que se generan contextos de vulnerabilidad $y$, por lo tanto, de asentamientos inseguros.

Las costas representan atractivos turísticos, industriales y urbanos para una mayor población que encuentra en estos lugares un foco de atracción para cumplir un sinnúmero de actividades y funciones. El caso de Acapulco, Guerrero, no escapó a esta dinámica cuyo detonante demográfico se presentó en las décadas del cincuenta y sesenta, al registrar un aumento importante en el número de habitantes. Este aumento de población provocó que poco a poco se fueran ocupando zonas que desde un principio no eran aptas para el desarrollo urbano, es decir, las nuevas viviendas se empezaron a asentar muy próximas a los cauces de arroyos y ríos, lo que ha provocado que se registren frecuentes eventos de desastre asociados a lluvias intensas.

El paso del huracán Pauline sobre las costas de Guerrero, representó en su momento el desastre asociado a un evento natural más importante en la historia reciente de Acapulco. Para 1997 la población y las autoridades quizá no dimensionaba los efectos de los huracanes cuando esta se asentaba en lugares inseguros, ya sean lugares próximos a la costa o sobre cauces de arroyos. Esto pudo representar un parteaguas en cuanto a la manera en que la sociedad considera, o no, un peligro inminente cuando no se han construido viviendas seguras o por la poca supervisión del desarrollo urbano que permitió la construcción en zonas no aptas.

El paso de la tormenta Manuel frente a las costas de Guerrero, si bien no tocó tierra en este estado, representó un mayor número de daños debido, entre otras muchas cosas, a que no se resolvieron los problemas de tipo urbano, a que no hubo acciones encaminadas a la prevención del riesgo y a la no reducción de los niveles de vulnerabilidad ya presentes desde 1997. De esta forma, el desastre es recurrente en la medida en que no se han podido reducir los factores asociados al riesgo ni a la reducción de los contextos de vulnerabilidad social, ambiental, económica e institucional.

El conocimiento sobre los fenómenos naturales es cada vez más preciso. El pronóstico y monitoreo en tiempo real de fenómenos como los huracanes se puede hacer hoy en día a través de diferentes plataformas electrónicas gracias a los satélites meteorológicos y a los sistemas de alerta temprana. A pesar de esto, los desastres son cada vez más frecuentes, pero lo más importante, cada vez son más costosos para la sociedad, ya sea en cuanto al número de muertos o en cuanto a las pérdidas económicas y materiales. Por lo tanto, se requiere profundizar en el conocimiento de los contextos específicos de vulnerabilidad para cada una de las comunidades que se encuentran 
expuestas a amenazas, pero además, se requiere socializar el conocimiento de estos estudios y la participación de esa sociedad expuesta.

El análisis de daños producidos por los dos ciclones abordados en este estudio permite entender de una manera general que la intensidad de los fenómenos intensos no esta directamente relacionada con el número de pérdidas o de daños sobre la sociedad expuesta, sino que son los contextos de vulnerabilidad o vulnerabilidades los que determinan de manera importante la magnitud del desastre. En este sentido, si los diferentes actores de la sociedad como las autoridades, científicos, tomadores de decisiones y la sociedad civil en general buscamos minimizar los daños ocasionados por cualquier fenómeno natural, debemos trabajar en cómo reducimos la vulnerabilidad y aumentamos la resiliencia de los asentamientos humanos para lograr que las ciudades sean más seguras ante los fenómenos naturales intensos en un contexto de cambio climático.

\section{Referencias}

1. Bitrán, D. (2001). Características del impacto socioeconómico de los principales desastres ocurridos en México en el periodo 1980-1999, México: Secretaría de Gobernación-Centro Nacional de Prevención de Desastres.

2. Cardona, O. D. (1996). Manejo ambiental y prevención de desastres: dos temas asociados. En María Augusta Fernández (Comp.), Ciudades en riesgo. Degradación ambiental, riesgos urbanos y desastres. (pp. 57-74). LA RED. En http://www.desenredando.org.

3. Cardona, O. D. (2001). La necesidad de repensar de manera holística los conceptos de vulnerabilidad y riesgo. Una crítica y una revisión necesarias para la gestión. Centro de Estudios sobre Desastres y Riesgos. Bogotá: CEDERI, Universidad de los Andes.

4. CONAGUA. (2009). Boletín Huracán Pauline, 5 de octubre-10 de octubre. México: Comisión Nacional del Agua, Subdirección General Técnica, Servicio Meteorológico Nacional. Recuperado de http://smn.conagua.gob.mx/ciclones/tempo1997/pacifico/pauline.pdf

5. CONAGUA. (1998). Resumen de la temporada de ciclones tropicales 1997. México: Comisión Nacional del Agua, Subdirección General Técnica, Servicio Meteorológico Nacional. Recuperado de http://smn1.conagua.gob.mx/ciclones/tempo1997/RTCT-1997.pdf

6. CONAGUA. (2012). Base de datos de ciclones tropicales que impactaron a México, 1970-2011, México: Comisión Nacional del Agua, Coordinación General del Servicio Meteorológico Nacional, Subgerencia de Pronóstico Meteorológico. Recuperado de http://smn.cna.gob.mx/ciclones/historia/ciclones1970-2011.pdf

7. CONAGUA. (2013). Reseña del huracán Manuel del Océano Pacífico. México: Comisión Nacional del Agua, Coordinación General del Servicio Meteorológico Nacional, Gerencia de Meteorología y Climatología-Subgerencia de Pronóstico Meteorológico. Recuperado de http://smn.conagua.gob.mx/ciclones/tempo2013/ pacifico/Manuel-p013.pdf

8. CONAGUA. (2016a). Temporada de ciclones 2015. México: Comisión Nacional del Agua, Servicio Meteorológico Nacional. Recuperado de http://smn.cna.gob.mx/index.php?option=com_content\&view=article\&i$d=276 \& \mid$ temid $=45$ 
9. CONAGUA. (2016b). Estación 12142. Acapulco de Juárez, Guerrero. México: Comisión Nacional del Agua, Servicio Meteorológico Nacional, Climatología estadística. Recuperado de http://smn.conagua.gob.mx/ index.php?option=com_content\&view=article\&id=182: guerrero\&catid=14: normales-por-estacion

10. CONAGUA. (2016c). Resumen de la temporada de ciclones tropicales del año 2013. México: Cirilo Bravo Lujano. Comisión Nacional del Agua, Servicio Meteorológico Nacional. Recuperado de http://smn.cna. gob.mx/tools/DATA/Ciclones\%20Tropicales/Resumenes/2013.pdf

11. DWUA. (2015). Demographia World Urban Areas. 11 Annual Edition, 2015. Recuperado de http://www. demographia.com/db-worldua.pdf

12. EM-Dat. (2016). The International Disaster Database. Belgium: Centre for Research on the Epidemiology of Disasters, Tormenta tropical Manuel. Número de fallecimientos. Recuperado de http://www.emdat.be/ country_profile/index.html

13. H. Ayuntamiento Constitucional de Acapulco de Juárez. (2015). Actualización del Plan Director de Desarrollo Urbano de Acapulco de Juárez, Guerrero. México: Secretaría de Desarrollo Urbano y Obras Públicas, Centro de Estudios de Urbanismo y Arquitectura S.A. de C.V. Recuperado de http://www.acapulco. gob.mx/transparencia/wp-content/uploads/marcojuridico/planes/PLAN_DIRECTOR_DE_DESARROLLO_ URBANO_2015.pdf

14. Gutiérrez, M. Y García, R. (1997), 121 muertos por Paulina en Oaxaca y Guerrero en La Jornada. Recuperado de http://www.jornada.unam.mx/1997/10/10/paulina.html

15. Lavell, T. A. (2002). Gestión de riesgos de desastre asociados con la variabilidad climática y el fenómeno ENSO en particular. Consideraciones generales y apuntes conceptuales. Documento de trabajo. IAI-LA RED.

16. Matías, L. G. (1998). Algunos efectos de la precipitación del huracán Paulina en Acapulco Guerrero. México: Investigaciones Geográficas, Boletín 37, 1998. UNAM.

17. Moseley, M. E. (1999). Convergent Catastrophe: Past Patterns and Future Implications of Collateral Natural Disasters in the Andes. En: Anthony Oliver-Smith y Susanna M. Hoffman (edits). The Angry Earth. Disaster in Anthropological Perspective. New York: Routledge, pp. 59-71.

18. Presidencia de la Nación. (2016). ¿Qué es comunicación de riesgo? Argentina: Ministerio de Salud. Recuperado de http://www.msal.gob.ar/salud-y-desastres/index.php/informacion-para-comunicadores/ique-es-comunicacion-de-riesgo

19. SEDESOL. (2004). Atlas de peligros naturales de la ciudad de Acapulco, Guerrero, México. Identificación y zonificación. México: Secretaría de Desarrollo Social-Habitat. Recuperado de http://www.acapulco.gob. $\mathrm{mx} /$ proteccioncivil/documentos/atlas2003.pdf

20. SEGOB. (2014). Impacto socioeconómico de los desastres en México durante 2013. México: Secretaría de Gobernación y Centro Nacional de Prevención de Desastres.

21. SEGOB. (2016a). Gastos de reconstrucción FONDEN 2013. México: Secretaría de Gobernación, Sistema Nacional de Protección Civil. Recuperado de http://www.proteccioncivil.gob.mx/work/models/ProteccionCivil/Resource/36/23/images/Copia\%20de\%20BUEN O\%20FONDEN\%20PARA\%20LA\%20WEB\%20 12\%20FEB\%2014\%20(2013).pdf 
22. SEGOB. (2016b). Gastos de reconstrucción FONDEN 2014. México: Secretaría de Gobernación, Sistema Nacional de Protección Civil, México. Recuperado de http://www. proteccioncivil.gob.mx/work/models/ ProteccionCivil/Resource/36/23/images/Copia\%20de\%20BUENO_FONDEN_EN_NUMEROS.pdf

23. SEGOB. (2016c). Boletín de alerta por ciclón tropical № 02. Tormenta tropical Manuel del Océano Pacífico. Secretaría de Gobernación. Sistema Nacional de Protección Civil. Recuperado de http://portal.proteccioncivil.gob.mx/upLoad/Alertamientos/2013/

24. SMN-CONAGUA. (2016). Glosario técnico. Servicio Meteorológico Nacional, Comisión Nacional el Agua. Recuperado de http://smn.cna.gob.mx/es/smn/glosario

25. Villegas, C. (2005). Recuperando el paraíso perdido: El proceso de reconstrucción en la ciudad de Acapulco. En: Virginia García Acosta (Coord.). La construcción social de riesgos y el huracán Paulina. México: Publicaciones de la Casa Chata, pp. 153-256.

26. Wilches-Chaux, G. (1993). La vulnerabilidad global. En: Andrew Maskrey (compilador). Los desastres no son naturales. LA RED. Recuperado de http://www.desenredando.org 